\title{
COMMENT \\ Packed red cell transfusion changes cerebral oxygenation and cardiac output
}

\author{
Heike Rabe ${ }^{1}$ \\ Pediatric Research (2019) 85:748-749; https://doi.org/10.1038/s41390-019-0294-y
}

During their hospital stay most very low birth weight infants develop anemia of prematurity and therefore require at least one packed red cell transfusion from adult donors. ${ }^{1,2}$ There is an ongoing debate about appropriate thresholds of hemoglobin values which indicate the need for a packed red cell transfusion. Concerns about effects of anemia on organ function, lowering of oxygen delivery to the tissues and long term consequences are discussed versus concerns of the parents regarding possible transfer of infectious diseases via the transfusion whilst the number of potential donors are declining. ${ }^{1,2}$ In an attempt to reduce the number of donors very-low birth weight infants are exposed to during their hospital stay, small satellite bags of packed red cells have been developed and the volume of one transfusion has been increased to $15 \mathrm{ml} / \mathrm{kg}$ body weight.

Jain et al. have used this volume of blood transfusion over a $3 \mathrm{~h}$ period in their prospective observational study and attempted to assess the changes to circulation assessed by electrical velocimetry and brain oxygenation measured by near infrared spectroscopy (NIRS). ${ }^{3}$ Their novel approach of calculating the oxygen delivery index from circulatory markers and brain oxygen saturation measured by NIRS has the potential to be used as an indicative marker for the need of a packed red cell transfusion. The study was not designed for capturing longer term neurodevelopmental outcomes for the premature infants less than 32 weeks gestation.

The NIRS method for measuring changes in brain oxygenation in preterm and term infants non-invasively has been used in research studies for decades. Recently large randomized controlled trials such as SafeBoosC II have been performed in order to establish its use in clinical practice for optimization of cerebral oxygenation for which normal values have not previously been established. ${ }^{4}$

More and more attention has turned to formal assessment of cardiac output, stroke volume, and superior vena cava flow as a surrogate marker for cerebral perfusion by using echocardiography performed by the attending neonatologist at the bedside. ${ }^{5}$ Such a clinical skill needs formal training and regular peer review. A recently published consensus papers can form the basis for a future European framework of practice. ${ }^{6}$ Measuring central circulatory parameters near to the heart might not be sufficient to assess poor circulatory status in the end-organ circulation. Thus other techniques such a tissue Doppler velocity or white light spectroscopy could provide additional information on perfusion and oxygenation in local tissues further away from the large vessels. ${ }^{7,8}$ Future studies should include techniques looking into the microcirculation of infants with non-invasive techniques.
The study by Jain et al. included preterm infants aged 3-48 days of life. During this period fetal hemoglobin is being replaced by the production of adult hemoglobin. It would be interesting to measure the oxygenation delivery index used in the study in preterm infants directly at birth and during the transition period of $\sim 72 \mathrm{~h}$ thereafter. Providing extra placental blood through either waiting before clamping and cutting the umbilical cord or by gently milking the umbilical cord towards the infant before cutting the cord provides a transfusion of fetal hemoglobin for the infant at birth with the benefit of improved survival rates. ${ }^{9}$ Neurodevelopmental outcome studies upto 3.5 years have not identified any adverse effect so far. ${ }^{10}$ It would be interesting to study whether the transfusion of fetal hemoglobin has a similar effect on oxygen delivery index in addition to observing changes of the index during the placental transfusion. Long term follow-up studies would be necessary to establish threshold values for the oxygen delivery index to be used for treatment decisions in the future.

\section{ADDITIONAL INFORMATION}

Competing interests: The authors declare no competing interests.

Publisher's note: Springer Nature remains neutral with regard to jurisdictional claims in published maps and institutional affiliations.

\section{REFERENCES}

1. ETTNO investigators. The 'effects of transfusion thresholds on neurocognitive outcome of extremely low birth-weight infants (ETTNO)' study: background, aims, and study protocol. Neonatology 101, 301-305 (2012).

2. Rabe, H., Fernandez Alvarez, J. R., Seddon, P., Lawn, C. \& Amess, P. N. A management guideline to reduce the frequency of blood transfusion in very low birth weight infants. Am. J. Perinat. 26, 179-83 (2009).

3. Jain, D et al. Effect of haemoglobin and cardiac output on cerebral oxygenation in preterm infants receiving packed red cell transfusion. Pediatr. Res. (2019).

4. Plomgaard, A. M. et al. TheSafeBoosC II randomized trial: treatment guided by near-infrared spectroscopy reduces cerebral hypoxia without changing early biomarkers of brain injury. Pediatr. Res. 79, 528-535 (2015).

5. De Boode, W. P. et al. The role of Neonatologist performed Echocardiography in the assessment and management of neonatal shock. Pediatr. Res. 84, 57-67 (2018).

6. Singh, Y. et al. Education, training, and accreditation of Neonatologist Performed Echocardiography in Europe - framework for practice. Pediatr. Res. 84, 13-17 (2018).

7. Nestaas, E. et al. Tissue doppler velocity imaging and event timings in neonates: a guide to image acquisition, measurement, interpretation, and reference values. Pediatr. Res. 84, 18-29 (2018). 
Packed red cell transfusion changes cerebral oxygenation and cardiac output H Rabe

8. Rabe, H. et al. Spectroscopic non-invasive measurement of hemoglobin compared with capillary and venous values in neonates. Neonatology 98, 1-5 (2010).

9. Katheria, A. et al. Placental transfusion-a review. J. Perinatol. 37, 105-111 (2017).
10. Rabe, H. et al. Neurodevelopmental outcomes at 2 and 3.5 years for very preterm babies enrolled to a randomized trial of milking the umbilical cord versus delayed cord clamping. Neonatology 109, 113-119 (2016). 